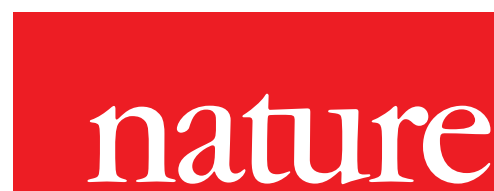

MASTERCLASSES

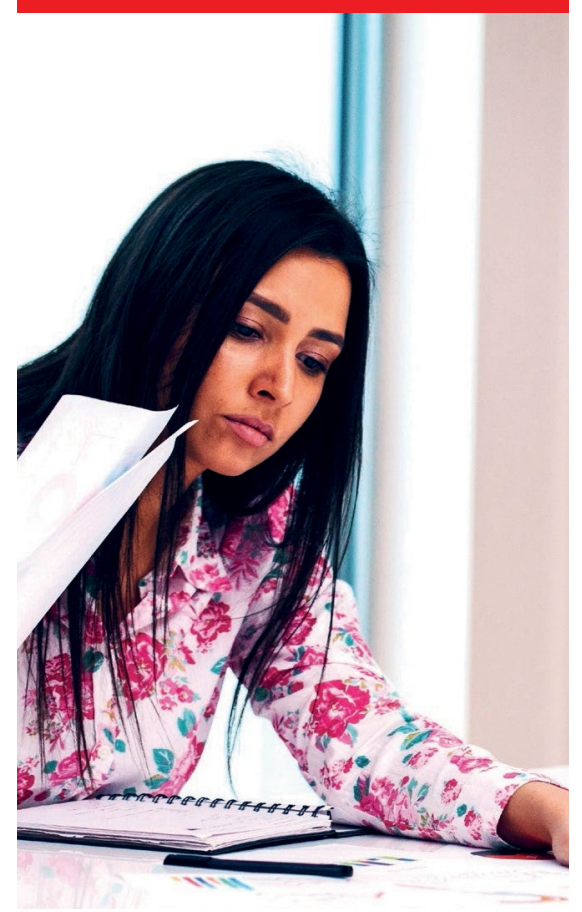

Free online

course for peer

reviewers

\section{For researchers new to \\ peer review or wishing \\ to develop their skills}

Register for free access at masterclasses.nature.com

Taught by Nature Research editors • 3-4 hours' learning • Free completion certificate

W masterclasses.nature.com

in Follow us on Linkedln

f Skills and Careers Forum for Researchers

\title{
A nanorotor for amplifying molecular movements
}

\author{
A DNA origami rotor amplifies and tracks DNA movements during \\ biochemical reactions, uncovering action mechanisms of genome- \\ processing enzymes.
}

( NA origami technology has unique advantages for creating $3 \mathrm{D}$ nanostructures with programmable configurations with molecular precision. Beyond generating distinct configurations of 3D nanostructures, researchers have started to explore the use of these nanostructures for various applications, ranging from imaging to biomedical science.

Xiaowei Zhuang, a biophysicist from Howard Hughes Medical Institute at Harvard University, and colleagues, use DNA nanorotors to amplify small rotational movements, thus enabling rotation-tracking of single biomolecules. They remark that "the DNA origami technology gave us the design freedom we needed to create rotors with desired properties to maximize responsivity".

The nanorotor the researchers designed resembles a ceiling fan. It has four blades that extend perpendicular to the rotation axis and possess a sufficient length to amplify the processive motion induced by biochemical reactions. To track their movements, the blades are labelled with fluorescent dyes for monitoring position trajectory.

The design of the "ceiling fan nanorotor" served its purpose; however, the researchers say, "we quickly realized that rotational amplification was not the only important feature to consider. In fact, any method to measure DNA rotation is fundamentally limited in its resolution by the extent and time scale of the Brownian motion."

To minimize the Brownian fluctuation, Zhuang and her collaborators modified the blades' design to maintain a low hydrodynamic drag and high torsional stiffness. The authors comment, "This is where the design possibilities of DNA origami came in handy" and "the dimensions of the rotors can easily be modified to suit other applications as needed".

In one example, the researchers showcased the use of origami-rotorbased imaging and tracking (ORBIT) by observing DNA unwinding events mediated by RecBCD helicase. They computed the unwinding rates based on the dye position trajectory. The ORBIT system offers an improved spatiotemporal
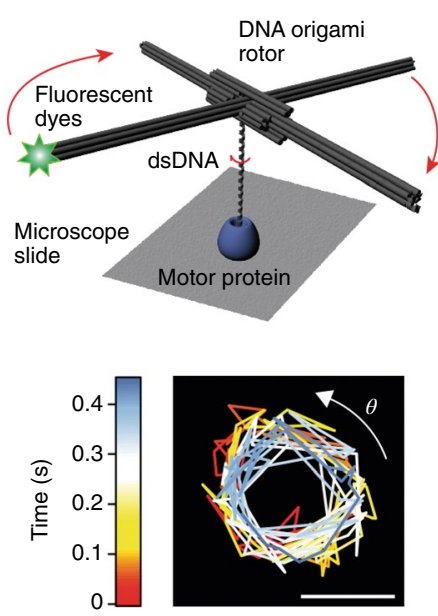

Top, the rotation of dsDNA induced by a motor protein is amplified by the DNA origami rotor. Bottom, position trajectory of the dye-labeled rotor blades. Figure reproduced from Kosuri, $\mathrm{P}$. et al. Nature 572, 136-140 (2019).

resolution compared to existing tools, and does not require any applied force. The team observed distinct stages of unwinding, pausing and backtracking; and they uncovered the mechanism of RecBCD initiation that involves reversible, ATPindependent unwinding of the first five base pairs at double-stranded DNA breaks.

In addition, the researchers applied ORBIT to study RNA polymerase (RNAP) activity during transcription. They directly tracked the rotational steps of single basepairs as RNAP rotates and translocates along the DNA helix.

Looking forward, "the team is exploring different uses of the DNA rotor, as well as different DNA origami structures specifically adapted for measurement of movements other than rotation."

\section{Lei Tang}

Published online: 30 August 2019 https://doi.org/10.1038/s41592-019-0556-y

Research paper

Kosuri, P. et al. Rotation tracking of genomeprocessing enzymes using DNA origami rotors. Nature 572, 136-140 (2019). 